GG erfüllt, oder es muss ein anderer Bundeskanzler gewählt werden, der eine ausreichende Mehrheit findet. ${ }^{66}$ Es ist kaum zu erwarten, dass das Selbstauflösungsrecht das Verhältnis vom Bundeskanzler zum Parlament zugunsten des Bundestages verändern würde. Selbstbewusste und fachkundige Mandatsträger sind gefragt. So selbstverständlich, wie die Abgeordneten im Alltagsgeschäft Fraktionsdisziplin als die notwendige Voraussetzung für das Funktionieren der parlamentarischen Demokratie anerkennen und ausüben, so verantwortungsvoll müssen sie in Situationen gefährdeter Handlungsfähigkeit auch die prinzipielle Unabhängigkeit nutzen, die ihnen das freie Mandat garantiert. Mit den Worten des Bundestagspräsidenten Norbert Lammert: „Im Bundestag schlägt das Herz der Demokratie oder es schlägt nicht. Das Parlament ist im Übrigen nicht Vollzugsorgan der Bundesregierung, sondern sein Auftraggeber."67

25. August 2005, Rdnr. 180; vgl. schon Volker Epping, in: Hermann v. Mangoldt/Friedrich Klein/ Christian Starck, Bonner Grundgesetz, 5. Auflage, München 2005, Art. 68 Rdnr. 24; Ute Mager, in: Ingo v. Münch / Philip Kunig, Grundgesetz, Kommentar, 5. Auflage, München 2004, Art. 68 Rdnr. 16.

66 Anders aber Volker Busse, a.a.O., S. 257, S. 259, der eine politische Beschädigung eines zurückgetretenen Kanzlers als Argument gegen die Forderung eines Rücktritts anführt.

67 Die Rede ist abrufbar unter http://www.bundestag.de/parlament/praesidium/reden/2005/013. html.

\title{
Struktur und Dauer der Gesetzgebungsverfahren des Bundes
}

\author{
Werner Reutter
}

Die „demokratie- und effizienzhinderlichen Verflechtungen“ zwischen Bund und Ländern provozieren nicht nur Reformstau und Politikblockaden, sondern führen auch zu „langwierigen und komplizierten Entscheidungsprozesse[n]“ - das zumindest war eines der zentralen Argumente, mit dem 2006 die Notwendigkeit einer Föderalismusreform begründet wurde. ${ }^{1}$ Im Entwurf zur Änderung des Grundgesetzes hieß es denn auch: „Bei der Gesetzgebung des Bundes haben die ausgeprägten Zustimmungsbefugnisse der Länder über den Bundesrat bei unterschiedlichen politischen Mehrheitsverhältnissen in Bund und Ländern immer wieder zu Verzögerungen oder sogar Verhinderung wichtiger Gesetzesvorhaben [...]

1 Deutscher Bundestag, BT-Drs. 16/813, Entwurf eines Gesetzes zur Änderung des Grundgesetzes (Artikel 22, 23, 33, 52, 72, 73, 74, 74a, 75, 84, 85, 87c, 91a, 91b, 93, 98, 104a, 104b, 105, 107, 109, 125a, 125b, 125c, 143c), 07.03.2006, in: http://dip.bundestag.de/btd/16/008/1600813.pdf (Stand: 20. April 2006), S. 7. Der Entwurf stammt von den Fraktionen der SPD und CDU/CSU und wurde nach Anhörungen mit den vom Rechtsausschuss empfohlenen Änderungen (BT-Drs. 16/2010) vom Bundestag in seiner 44. Sitzung am 30. Juni 2006 angenommen. Der Bundesrat stimmte am 7. Juli 2006 dem Gesetzespaket zu. 
geführt, ..."2 Die Diagnose impliziert die Therapie: Wenn Verflechtung das Problem ist, ist Trennung die Lösung. ${ }^{3}$ Die vorgenommene Verfahrensentflechtung insbesondere mittels einer Reduzierung der Anzahl zustimmungspflichtiger Gesetze und einer Neuverteilung der legislativen Kompetenzen soll daher die „Handlungs- und Entscheidungsfähigkeit von Bund und Ländern verbessern", was - zumindest implizit - die Beschleunigung der Gesetzgebungsverfahren einschließt.

Selbst wenn man die demokratie- und föderalismustheoretischen Dimensionen der beschlossenen Grundgesetzänderungen beiseitelässt, überrascht, dass eine Bestandsaufnahme und Analyse der Dauer von Gesetzgebungsverfahren in der Bundesrepubik Deutschland weitgehend fehlt. Einzig Thomas König und Thomas Bräuninger haben im Rahmen einer Untersuchung der Gesetzgebung im Föderalismus über eine Ereignisdatenanalyse auch „Determinanten der Verabschiedungsdauer“ ermittelt. ${ }^{4}$ Ihr Erkenntnisinteresse liegt allerdings weniger darin, die zeitliche Struktur von Gesetzgebungsverfahren zu analysieren. Vielmehr wollen sie die Faktoren herausarbeiten, die die „Überlebenswahrscheinlichkeit“ von Gesetzesinitiativen bestimmen. Aufgrund ihrer Erhebungen kommen sie unter anderem zu folgenden Ergebnissen: Nach circa 350 Tagen ist die Hälfte der Gesetzesvorschläge verabschiedet worden, die Bundesregierung konnte ihre Vorhaben am effizientesten, das heißt am schnellsten realisieren, und die Unterscheidung von Zustimmungs- und Einspruchsgesetzen schlug sich „zwar nicht in der Gesamtschau, aber in einzelnen diskreten Phasen der Gesetzgebung " nieder.5 Doch ändert diese Einzelstudie nichts an dem generellen Befund: Die Langwierigkeit von parlamentarischen Entscheidungsprozessen scheint eher „gefühlt" als wissenschaftlich verifiziert.

Das ist umso bedauerlicher, als die folgende Analyse für die 15. Wahlperiode sowie von „Schlüsselentscheidungen“ der 7. bis 15. Wahlperiode des Bundestages eine Reihe von immer wieder beklagten Problemen zumindest relativiert. Insgesamt lässt sich dabei feststellen, dass die mit der Föderalismusreform beschlossenen Änderungen im Bereich der Gesetzgebungskompetenzen sich mit dem Argument, der hohe Anteil zustimmungspflichtiger Gesetze verzögere parlamentarische Entscheidungsprozesse, kaum rechtfertigen lässt. Eben-

2 Deutscher Bundestag, BT-Drs. 16/2010, S. 3; dieselbe Formulierung findet sich in dem Entwurf, BT-Drs. 16/813, a.a.O., S. 1.

3 Fritz W. Scharpf, Thesenpapier zur Anhörung des Rechtsausschusses zum Thema „Föderalismusreform" am 15./16. Mai 2006, in: http://www.bundestag.de/aussschüsse/a06/foederalismusreform/Anhoerung/01_Allgemeiner_Teil/Stellungnahmen/Prof_Dr_Fritz_W_Scharpf.pdf (Stand: 16. Mai 2006), S. 6; vgl. auch: Werner Reutter, Regieren nach der Föderalismusreform, in: APuZ, B 50 (2006), S. 12 - 17; ders., Föderalismusreform und Gesetzgebung, in: ZPol, 16. Jg. (2006), S. $1249-1274$.

4 Thomas König / Thomas Bräuninger, Gesetzgebung im Föderalismus, Speyerer Forschungsberichte 237, Hochschule für Verwaltungswissenschaften Speyer 2005; vgl. neuerdings auch: Simone Burkhart / Philip Manow, Was bringt die Föderalismusreform? Wahrscheinliche Effekte der geänderten Zustimmungspflicht, MPIFG Working Paper 06/6, Köln 2006, in: http://www.mpifg. de/pu/workpap/wp06-6/wp06-6.html (Stand: 6. Oktober 2006), S. 12 ff. Auch wird in dem von Peter Schindler herausgegebenen Datenhandbuch zum Deutschen Bundestag die Dauer von Gesetzgebungsverfahren bis zur 12. Wahlperiode ausgewiesen, Peter Schindler (Hrsg.), Datenhandbuch zur Geschichte des Deutschen Bundestages 1949 bis 1999, Baden-Baden 1999, S. 3702 f.; dies wurde jedoch in dem aktuellen Datenhandbuch nicht weitergeführt, Michael F. Feldkamp unter Mitarbeit von Birgit Ströbel, Datenhandbuch zur Geschichte des Deutschen Bundestages 1994 bis 2003, Baden-Baden 2005.

5 Thomas König/ Thomas Bräuninger, a.a.O., S. 62, S. 68 f. 
so wenig scheinen die verabschiedeten Maßnahmen geeignet, das Gesetzgebungsverfahren entscheidend zu verkürzen. Insbesondere drei Aspekte sind dabei von Bedeutung: erstens die Frage, ob eine Zustimmungspflichtigkeit die Verabschiedung von Gesetzen verzögert. Zweitens ist zu prüfen, ob die Anrufung des Vermittlungsausschusses Gesetzgebungsverfahren über die Maßen verlängert. Schließlich ist herauszuarbeiten, inwiefern sich bei Schlüsselentscheidungen, die politisch zumeist kontrovers diskutiert wurden, Entscheidungsverfahren als besonders langwierig gestalteten.

\section{GESTA und „Schlüsselentscheidungen": methodische Vorbemerkungen}

Die für eine Analyse der Dauer von Gesetzgebungsverfahren erforderlichen Daten sind einfach, wenn auch aufwendig, zu erschließen. Seit 1973 erstellen Bundestag und Bundesrat, Gruppe Datenverarbeitung, gemeinsam eine Dokumentation zum Stand der Gesetzgebung: GESTA. Diese Dokumentation erschien bis 2002 als Loseblattsammlung, die nach Ende der jeweiligen Wahlperiode in einem Abschlussband zusammengefasst und, ergänzt durch Übersichten und Verzeichnisse, veröffentlicht wurde. ${ }^{6}$ Seit der 14 . Wahlperiode sind die Daten online verfügbar, und mit Beginn der 15. Wahlperiode wurden Loseblattsammlung und deren Veröffentlichung in einem Abschlussband eingestellt; die Daten werden seitdem nur noch online, als Teil des Dokumentations- und Informationssystems für parlamentarische Vorgänge (DIP) der Öffentlichkeit zur Verfügung gestellt. ${ }^{7}$ In dem aktuellen Datensatz ist jeder in den Bundestag oder Bundesrat eingebrachte Gesetzentwurf einer Sachgruppe zugeordnet, die sich in der Regel an dem federführenden Ressort orientiert (ausnahmsweise am federführenden Bundestagsausschuss). Aus der Kombination von Sachgruppenkennzeichnung ${ }^{8}$ und Nummerierung ergibt sich die „GESTA-Nummer“. Für jeden Entwurf weist GESTA folgende Detailinformationen nach:

- Sachgruppe und Sachgruppennummer (= GESTA-Nummer, zum Beispiel: B. Inneres - B003),

- Sachgebiet (zum Beispiel 26),

- Datum der Verkündung, der Ablehnung, der Rücknahme, einer Nichtigkeitserklärung durch das Bundesverfassungsgericht oder einer anderweitigen Erledigung,

- Hauptschlagwort (zum Beispiel: Zuwanderungsgesetz),

- vollständiger Titel des Gesetzes (zum Beispiel Gesetz zur Steuerung der Zuwanderung und zur Regelung des Aufenthalts und der Integration von Unionsbürgern und Ausländern [Zuwanderungsgesetz]),

6 Deutscher Bundestag / Deutscher Bundesrat (Hrsg.), Stand der Gesetzgebung des Bundes. Verkündete und nicht Gesetz gewordene Entwürfe. Abschlussbände 7. bis 14. Wahlperiode, BadenBaden 1977, 1981, 1983, 1987, 1991, 1995, 1999, 2003; für die 15. Wahlperiode vgl. http:// dip.bundestag.de/GESTA.online.15.pdf (Stand: 26. April 2006).

7 Vgl. zum Vorstehenden die Benutzerhinweise in einem der Abschlussbände oder in: http://dip. bundestag.de/gesta/15/benutzerhinweise.pdf (Stand: 26. April 2006).

8 Für die 15. Wahlperiode waren folgende Sachgruppen aufgeführt: A - Äußeres, B - Inneres, C Justiz, D - Finanzen, E - Wirtschaft und Arbeit, F - Verbraucherschutz, Ernährung und Landwirtschaft, G - Gesundheit und soziale Sicherung, H - Verteidigung, I - Familie, Senioren, Frauen und Jugend, J - Verkehr, Bau- und Wohnungswesen, K - Bildung und Forschung, M Kultur, N - Umwelt, Naturschutz und Reaktorsicherheit. Bei völkerrechtlichen Verträgen wird den Ordnungsnummern ein „X“ vorangestellt, also zum Beispiel „XB“ (= völkerrechtliche Verträge zum Bereich Inneres). Für die Ressortgliederung zwischen der 1. bis 12. WP vgl. Peter Schindler, a.a.O., S. 2408 ff.; für die 13., 14. und 15. WP vgl. Michael F. Feldkamp / Birgit Ströbel, a.a.O., S. $278-281$. 
- Initiative (von wem eingebracht bei Bundesrat oder Bundestag),

- Zustimmungsbedürftigkeit (ja / nein, abweichende Meinung des Bundesrates),

- europäische Impulse,

- Bezüge (zu anderen Vorlagen, zu Urteilen des Bundesverfassungsgerichts, zu Koalitionsvereinbarungen, zu Berichten von Ministerien etc.),

- kurze Beschreibung des Inhalts (auf ein bis zwei Seiten),

- Gang der Gesetzgebung in Bundesrat und Bundestag (einschließlich Überweisung an Ausschüsse, Änderungsanträge, Anrufung des Vermittlungsausschusses usw.),

- Datum des Gesetzes, Verkündungsdatum, Datum des Inkrafttretens und Fundstelle im Bundesgesetzblatt.

Für die vorliegende Analyse wurden folgende Daten erhoben:

- Sachgruppe und Sachgruppennummer,

- Zustimmungspflichtigkeit (ja / nein, so wie im verkündeten Gesetz ausgewiesen),

- Anrufung des Vermittlungsausschusses (ja / nein),

- Tag der Einbringung des Gesetzentwurfes in den Bundesrat (= Datum der Drucksache des Bundesrats),

- Tag der Einbringung des Gesetzentwurfes in den Bundestag (= Datum der Drucksache des Bundestags),

- 1. Beratung Bundestag,

- 3. Beratung Bundestag (oder Tag der Schlussabstimmung),

- Datum der Verkündung.

GESTA hat sich nach seiner Etablierung rasch als die zentrale Quelle für entsprechende Dokumentationen und wissenschaftliche Untersuchungen über die Gesetzgebung entwickelt $^{9}$, und viele davon sind in der Zeitschrift für Parlamentsfragen erschienen. ${ }^{10}$ Doch hat Helmuth Schulze-Fielitz bereits 1986 auf „Fallstricke der Gesetzgebungsstatistik“ vor allem im Hinblick auf deren Verwendbarkeit für eine Gesetzgebungswissenschaft aufmerksam gemacht. Die GESTA-Dokumentation ist primär eine Verlaufsstatistik. Sie dokumentiert den formalen Gesetzgebungsprozess, blendet also vorparlamentarische Phasen von Entscheidungsprozessen aus, gibt keine Anhaltspunkte über die unterschiedliche Bedeutung gesetzlicher Regelungen und weist textidentische Entwürfe aus der Mitte des Bundestages und der Bundesregierung gesondert aus. ${ }^{11}$ Diese „Fallstricke“ sind zweifellos bei jeder Analyse der Gesetzgebung zu beachten und verlangen vor allem qualitative Substanziierungen von quantitativen Aussagen, die auf der GESTA beruhen. So sagt ein noch so hoher Anteil von am

9 Erwähnt seien nur die von Peter Schindler, a.a.O., und von Michael F. Feldkamp / Birgit Ströbel, a.a.O., herausgegebenen Datenhandbücher zur Geschichte des Deutschen Bundestages.

10 Erwähnt sei zum Beispiel die kontinuierliche Parlamentsstatistik von zuletzt: Michael F. Feldkamp, Deutscher Bundestag 1987-2005: Parlaments- und Wahlstatistik, in: ZParl, 37. Jg. (2006), H. 1, S. 3 - 19; oder Christian Dästner, Zur Entwicklung der Zustimmungsbedürftigkeit von Bundesgesetzen seit 1949, in: ZParl, 32. Jg. (2001), H. 2, S. 290 - 309.

11 Helmuth Schulze-Fielitz, Fallstricke der Gesetzgebungsstatistik, in: Zeitschrift für Gesetzgebung, 1. Jg. (1986), S. 364 - 368. Schulze-Fielitz erwähnt noch zwei weitere Aspekte: So wird die Bedeutung der Ausschussarbeit im Bundestag überbewertet, wenn allein die Anzahl von Änderungsvorschlägen, die auf Initiativen der Ausschüsse zurückgehen, gezählt werden. Denn diese Vorschläge beziehen sich vielfach auf formale Aspekte (wie Daten, Fristen etc.) und sagen nichts über den Einfluss aus, den die Ausschüsse inhaltlich ausüben. Auch die Angaben zum Abstimmungsverhalten im Bundestag sind nicht unproblematisch und erlauben zum Beispiel keineswegs ohne weiteres Aussagen über konsensual oder kontrovers verabschiedete Gesetze. 
Bundesrat gescheiterten Gesetzen wenig aus über die Reformfähigkeit in Deutschland, wenn nicht geprüft ist, um welche Art von Gesetzen es sich dabei gehandelt hat. Für die vorliegende Untersuchung sind diese Einwände allerdings - von einigen Aspekten abgesehen vernachlässigbar, denn das Erkenntnisinteresse deckt sich weitgehend mit dem Zweck der GESTA-Dokumentation. Eine Verlaufsstatistik bietet eine solide Datengrundlage für eine Analyse der Dauer von Gesetzgebungsverfahren, abgesehen allerdings von dem Umstand, dass auch hier die Frage zu berücksichtigen ist, ob sich zwischen gesetzlichen Regelungen unterschiedlichen Gewichts systematische Differenzen bei der Dauer ergeben haben.

\begin{tabular}{|c|c|c|c|c|c|c|c|c|c|c|c|}
\hline \multicolumn{12}{|c|}{ Tabelle 1: Schlüsselentscheidungen des Bundestages (Anzahl der Gesetze) } \\
\hline Wahlperiode & 1.-6. & 7. & 8. & 9. & 10. & 11. & 12. & 13. & 14. & 15. & Tesamt \\
\hline nach Klaus von Beyme & 80 & 16 & 11 & 5 & 8 & 12 & 18 & - & - & - & 150 \\
\hline nach König/Bräuninger ${ }^{2}$ & - & - & 14 & 7 & 11 & 18 & 20 & 16 & 15 & - & 101 \\
\hline nach Datenhandbuch & 94 & 26 & 11 & 5 & 10 & 15 & 16 & - & - & - & 177 \\
\hline eigene Ergänzungen & - & 26 & 11 & 5 & 10 & 15 & 16 & 18 & 14 & 23 & 138 \\
\hline \multicolumn{12}{|c|}{$\begin{array}{l}\text { a "Wichtige“ Gesetzesinitiativen. } \\
\text { Quellen: Klaus von Beyme, Der Gesetzgeber. Der Bundestag als Entscheidungszentrum, Opladen 1997, } \\
\text { S. 63; Peter Schindler (Hrsg.), a.a.O, S. } 2597 \text { - 2635, Thomas König / Thomas Bräuninger, a.a.O., S. 32; } \\
\text { eigene Ergänzungen auf Grundlage von GESTA. }\end{array}$} \\
\hline
\end{tabular}

Es existieren diverse Versuche, Gesetze nach ihrer inhaltlichen und politischen Relevanz zu hierarchisieren (vgl. Tabelle 1). Um die Rolle des Bundestages als Gesetzgeber zu untersuchen, hat zum Beispiel Klaus von Beyme für die 1. bis 12. Wahlperiode insgesamt $150 \mathrm{Ge-}$ setze als „Schlüsselentscheidungen“ bestimmt und Politikfeldspezialisten „zur Kontrolle“ vorgelegt. Beyme hat sein Sample „nach einigen Kriterien ausgewählt“, die ihm „konsensfähig" schienen. ${ }^{12}$ Er nennt dabei: (1) die Breite des Interesses, die die Entscheidung fand, (2) die erzielte Tiefe und Dauerhaftigkeit der Regelung und (3) die Konflikthaftigkeit der Entscheidung. Doch trotz der methodisch skrupulösen Herangehensweise Beymes kann eine solche Liste naturgemäß nie ganz frei sein von subjektiven Wertungen. So hat Peter Schindler die Liste ergänzt, in der 12. Wahlperiode um zwei Gesetze gekürzt und statt 150 insgesamt 177 Gesetze, die zwischen 1949 und 1994 verkündet wurden, als Schlüsselentscheidungen qualifiziert. ${ }^{13}$

12 Klaus von Beyme, Der Gesetzgeber. Der Bundestag als Entscheidungszentrum, Opladen 1997, S. 68.

13 Peter Schindler, a.a.O., S. 2597 - 2635. Helmuth Schulze-Fielitz hat eine eigene Typologie entwickelt und neben Ratifikations- und Kooperationsgesetzen, die auf völkerrechtliche Vereinbarungen zurückgehen und mit einem Regierungsvorbehalt versehen sind, Kodifikations- von Anpassungsgesetzen unterschieden. Während Erstere einen „wesentlichen“ Teilbereich „in sich schlüssig und abschließend" ordnen, knüpfen Letztere an eine bestehende Gesetzeslage an und ändern diese teilweise. Streng genommen, kann in der 9. Wahlperiode lediglich das Künstlersozialversicherungsgesetz als „kodifikatorische[s] Reformgesetz" betrachtet werden, die restlichen Kodifikationsgesetze in dieser Legislaturperiode - Schulze-Fielitz nennt sieben Gesetze - „ergänzen“ und „modifizieren“ bereits bestehende rechtliche Regelungen ,in einem Ausmaß, daß man von einem ,neuen' Gesetz sprechen muß“. Helmuth Schulze-Fielitz, Theorie und Praxis parlamentarischer Gesetzgebung - besonders des 9. Deutschen Bundestages (1980-1983), Berlin 1988, S. 89, S. 95. 
Die Einordnung von Schindler war Ausgangspunkt für die vorliegende Untersuchung, sie wurde jedoch um Schlüsselentscheidungen aus der 13., 14. und 15. WP ergänzt (vgl. Tabelle 2). ${ }^{14}$ Die im Weiteren insgesamt 138 herangezogenen Schlüsselentscheidungen enthalten zweifellos manches Gesetz, dessen Relevanz sich nicht jedem unmittelbar aufdrängt, gleichzeitig dürften Gesetze fehlen, die viele für wichtig halten. Vermutlich sind Entscheidungen überrepräsentiert, die Konflikte hervorgerufen und politisch umstritten waren, also breiten Raum in den Medien und damit in der Aufmerksamkeit des Autors gefunden haben. Diese Schwäche der hier ausgewählten Entscheidungen wird jedoch dadurch gemildert, dass der Zweck der Auswahl darin besteht, die Verfahrensdauer zu analysieren. Es war also nicht, zumindest nicht nur, die Relevanz des Gesetzes für das jeweilige Politikfeld ausschlaggebend, sondern die Intensität der Auseinandersetzungen, die das Gesetzgebungsverfahren begleitet haben. Aus dieser Perspektive mag eine Liste von Schlüsselentscheidungen, die politisch umstrittene Gesetze beinhaltet, sogar besonders aussagekräftig sein. Deswegen sind auch zwei Gesetze enthalten, die nach Verkündung vom Bundesverfassungsgericht für nichtig erklärt wurden (das Zuwanderungsgesetz von 2002 und das Gesetz zum europäischen Haftbefehl).

\begin{tabular}{|c|c|c|c|}
\hline & $\begin{array}{c}\text { Tag der } \\
\text { Einbringung } \\
\text { des Gesetzes in } \\
\text { Bundestag } \\
\end{array}$ & $\begin{array}{l}\text { Tag der Ver- } \\
\text { abschiedung } \\
\text { im Bundestag }\end{array}$ & $\begin{array}{l}\text { Tag der Ver- } \\
\text { kündung } \\
\text { im BGBl }\end{array}$ \\
\hline \multicolumn{4}{|l|}{ 13. Wahlperiode (1994 bis 1998) } \\
\hline Jugoslawien-Strafgerichtshof-Gesetz & 29.11 .1994 & 09.03 .1995 & 13.04 .1995 \\
\hline Ruanda-Strafgerichtshof-Gesetz & 12.06.1997 & 05.02 .1998 & 08.05 .1998 \\
\hline 33. Strafrechtsänderungs-Gesetz ( $\$ \$ 177$ ff.) & 21.03.1997 & 15.05.1997 & 04.07.1997 \\
\hline Euro-Einführungsgesetz & 04.12 .1997 & 02.04 .1998 & 15.06.1998 \\
\hline GG-Änderung (Art. 3, Post- und Fernmeldegeheimnis) & 01.10 .1997 & 16.01 .1998 & 31.03 .1998 \\
\hline Jahressteuergesetz 1996 & 27.03 .1995 & 02.06 .1995 & 20.10 .1995 \\
\hline Jahressteuergesetz 1997 & 11.06 .1996 & 07.11 .1996 & 27.12 .1996 \\
\hline Energiewirtschaft & 23.03.1997 & 28.11.1997 & 06.03 .1998 \\
\hline Arbeitnehmer-Entsendegesetz & 25.09 .1995 & 30.11 .1995 & 29.02 .1996 \\
\hline 2. Stufe Pflegeversicherung & 16.02 .1996 & 10.05 .1996 & 07.06 .1996 \\
\hline Europäische Betriebsräte & 06.05 .1996 & 27.09 .1996 & 31.10 .1996 \\
\hline Rentenreformgesetz 1999 & 24.06 .1997 & 10.10 .1997 & 22.12.1997 \\
\hline Telekommunikationsgesetz & 23.04 .1996 & 13.06.1996 & 31.07 .1996 \\
\hline Postgesetz & 30.05 .1997 & 09.10 .1997 & 30.12 .1997 \\
\hline HIV-Hilfegesetz & 09.05 .1995 & 29.06 .1995 & 28.07.1995 \\
\hline 1. GKV-Neuordnungsgesetz & 08.10 .1996 & 15.11 .1996 & 30.06 .1997 \\
\hline 2. GKV-Neuordnungsgesetz & 12.11.1996 & 20.03.1997 & 30.06 .1997 \\
\hline Europol-Gesetz & 10.04 .1997 & 10.10 .1997 & 19.12.1997 \\
\hline
\end{tabular}

14 Thomas König / Thomas Bräuninger, a.a.O., haben - auf Grundlage der von Beyme entwickelten Kriterien - „wichtige“ Gesetzesinitiativen für die 8. bis 14. Legislaturperiode bestimmt. Sie kommen, da sie auch gescheiterte Gesetzentwürfe einbeziehen, naturgemäß zu höheren Zahlen. 


\begin{tabular}{|c|c|c|c|}
\hline \multicolumn{4}{|l|}{ Fortsetzung Tabelle 2: } \\
\hline & $\begin{array}{c}\text { Tag der } \\
\text { Einbringung } \\
\text { des Gesetzes in } \\
\text { Bundestag } \\
\end{array}$ & $\begin{array}{l}\text { Tag der Ver- } \\
\text { abschiedung } \\
\text { im Bundestag }\end{array}$ & $\begin{array}{l}\text { Tag der Ver- } \\
\text { kündung } \\
\text { im BGBl }\end{array}$ \\
\hline \multicolumn{4}{|l|}{ 14. Wahlperiode (1998 bis 2002) } \\
\hline Staatsangehörigkeitsrecht & 16.03.1999 & 07.05.1999 & 23.07.1999 \\
\hline Stiftung „Erinnerung, Verantwortung und Zukunft“ & 26.05 .2000 & 06.07 .2000 & 11.08 .2000 \\
\hline Professorenbesoldungsreform & 31.08 .2001 & 09.11 .2001 & 22.02 .2002 \\
\hline Zuwanderungsgesetz & 14.01 .2002 & 01.03 .2002 & 25.06.2002 \\
\hline Lebenspartnerschaftsgesetz & 04.07 .2000 & 10.11 .2000 & 22.02.2001 \\
\hline Steuerentlastungsgesetz 1999/2000/2002 & 09.11 .1998 & 04.03 .1999 & 31.03.1999 \\
\hline Ökologische Steuerreform & 17.11 .1998 & 03.03 .1999 & 29.03.1999 \\
\hline Steuersenkungsgesetz & 30.03 .2000 & 18.05.2000 & 26.10 .2000 \\
\hline Maßstäbegesetz & 07.05 .2001 & 05.07 .2001 & 12.09.2001 \\
\hline BSE-Maßnahmegesetz & 06.02 .2001 & 15.02 .2001 & 21.02.2001 \\
\hline Sozialversicherung & 17.11.1998 & 10.12 .1998 & 28.12.1998 \\
\hline Geringfügige Beschäftigung & 19.01.1999 & 04.03 .1999 & 29.03.1999 \\
\hline Prostitutionsgesetz & 08.05 .2001 & 19.10 .2001 & 27.12 .2001 \\
\hline GKV-Gesundheitsreformgesetz 2000 & 23.06.1999 & 04.11 .1999 & 29.12 .1999 \\
\hline \multicolumn{4}{|l|}{ 15. Wahlperiode (2002 bis 2005 ) } \\
\hline Zuwanderungsgesetz & 07.02 .2003 & 09.05 .2003 & 05.08 .2004 \\
\hline Informationsfreiheitsgesetz & 14.12 .2004 & 03.06 .2005 & 13.09.2005 \\
\hline Terrorismusbekämpfung & 08.04 .2003 & 17.10 .2003 & 27.12 .2003 \\
\hline Europäisches Haftbefehlsgesetz & 15.10 .2003 & 11.03.2004 & 26.07.2004 \\
\hline Gewerbesteuergesetz & 08.09 .2003 & 17.10 .2003 & 29.12 .2003 \\
\hline Haushaltsbegleitgesetz & 08.09 .2003 & 17.10 .2003 & 31.12 .2003 \\
\hline Alterseinkünftegesetz & 26.02 .2004 & 29.04 .2004 & 09.07.2004 \\
\hline $\begin{array}{l}\text { Erstes Gesetz für moderne Dienstleistungen } \\
\text { am Arbeitsmarkt }\end{array}$ & 05.11 .2002 & 15.11 .2002 & 30.12 .2002 \\
\hline $\begin{array}{l}\text { Zweites Gesetz für moderne Dienstleistungen } \\
\text { am Arbeitsmarkt }\end{array}$ & 05.11 .2002 & 15.11 .2002 & 30.12 .2002 \\
\hline Gesetz zu Reformen am Arbeitsmarkt & 02.09 .2003 & 26.09 .2003 & 30.12 .2003 \\
\hline $\begin{array}{l}\text { Drittes Gesetz für moderne Dienstleistungen } \\
\text { am Arbeitsmarkt }\end{array}$ & 01.10 .2003 & 17.10 .2003 & 27.12 .2003 \\
\hline $\begin{array}{l}\text { Viertes Gesetz für moderne Dienstleistungen } \\
\text { am Arbeitsmarkt }\end{array}$ & 05.09 .2003 & 17.10 .2003 & 29.12 .2003 \\
\hline Zwölftes SGB V-Änderungsgesetz & 05.11 .2002 & 15.11 .2002 & 17.06.2003 \\
\hline Beitragssatzsicherungsgesetz & 05.11 .2002 & 15.11 .2002 & 30.12 .2002 \\
\hline $\begin{array}{l}\text { Gesetz zur Einordnung des Sozialhilferechts in das } \\
\text { Sozialgesetzbuch }\end{array}$ & 05.09 .2003 & 17.10 .2003 & 30.12 .2003 \\
\hline GKV-Modernisierungsgesetz & 08.09 .2003 & 26.09 .2003 & 19.11.2003 \\
\hline $\begin{array}{l}\text { Zweites Gesetz zur Änderung des Sechsten Buches } \\
\text { Sozialgesetzbuch und anderer Gesetze }\end{array}$ & 23.10 .2003 & 06.11 .2003 & 30.12 .2003 \\
\hline RV-Nachhaltigkeitsgesetz & 09.12 .2003 & 11.03 .2004 & 26.07.2004 \\
\hline Kinder-Berücksichtigungsgesetz & 03.09 .2004 & 01.10 .2004 & 20.12 .2004 \\
\hline Autobahnmautgesetz für schwere Nutzfahrzeuge & 06.09 .2004 & 01.10 .2004 & 07.12 .2004 \\
\hline 7. HRGÄndG & 28.08.2003 & 01.07 .2004 & 03.09 .2004 \\
\hline Errichtung der Akademie der Künste (AdKG) & 16.06 .2004 & 11.11 .2004 & 09.05 .2005 \\
\hline $\begin{array}{l}\text { Änderung dienst- und arbeitsrechtlicher Vorschriften } \\
\text { im Hochschulbereich (HdaVÄndG) }\end{array}$ & 09.11 .2004 & 03.12 .2004 & 30.12 .2004 \\
\hline
\end{tabular}




\section{Dauer der Gesetzgebung im Bundesstaat: rechtliche Rahmenbedingungen}

Grosso modo lässt sich das Gesetzgebungsverfahren in vier Stadien untergliedern, deren Dauer teilweise durch das Grundgesetz und die Geschäftsordnung des Bundestages (GOBT) beschränkt wird. ${ }^{15}$ Grundlegend ist dabei das Diskontinuitätsprinzip, demgemäß Parlamentsvorlagen mit Ende einer Legislaturperiode verfallen. Allerdings können Gesetzentwürfe, die vor der konstituierenden Sitzung eines neu gewählten Bundestages in den Bundesrat eingebracht wurden, in der laufenden Legislaturperiode behandelt und entschieden werden. Dieses Diskontinuitätsprinzip ist für verkürzte Wahlperioden von besonderer Bedeutung. Allerdings bestehen darüber hinaus nur wenige rechtliche Vorgaben, die das Gesetzgebungsverfahren zeitlich strukturieren könnten. Im Weiteren werden nur die Normen für den Regelfall dargelegt. Die Veränderungen im Zeitablauf und die vielfältigen Ausnahmen (bei eilbedürftigen Gesetzen, bei Haushaltsgesetzen, bei EU-Vorlagen, bei GGÄnderungen usw.) werden nur gelegentlich berücksichtigt.

(1) Vorverfahren: Der so genannte 1. Durchgang erfasst das Vorverfahren im Bundesrat (Art. 76 Abs. 2 und 3 GG), das zwei Typen kennt: zum einen die Einbringung eines Entwurfs durch ein Land (oder durch mehrere Länder) und die anschließende Stellungnahme durch die Bundesregierung; zum anderen Regierungsentwürfe, die zur Stellungnahme an den Bundesrat geleitet werden (Art. 76 GG). Der Bundesrat hat das Recht, innerhalb von sechs Wochen eine Stellungnahme zu einer Vorlage der Regierung abzugeben (bei als eilbedürftig bezeichneten Gesetzen sind es drei Wochen). ${ }^{16}$ Nach Ablauf dieser Frist kann die Regierung den Entwurf in den Bundestag einbringen und die Stellungnahme des Bundesrates nachreichen. Bei Vorlagen des Bundesrates ist die Bundesregierung verpflichtet, den Entwurf innerhalb von sechs Wochen dem Bundestag zuzuleiten (bei besonders eilbedürftigen Vorlagen sind es drei Wochen).

(2) Verfahren im Bundestag: Nach Ende des Vorverfahrens - beziehungsweise bei Entwürfen aus der Mitte des Bundestages ohne Vorverfahren - folgen die parlamentarischen Beratungen im Bundestag (Art. 77 Abs. 1 GG). In aller Regel umfassen diese drei Lesungen, ausgenommen sind völkerrechtliche Verträge, über die zumeist bei der zweiten Lesung eine Schlussabstimmung erfolgt. Sieht man von dem erwähnten Diskontinuitätsprinzip ab, ergeben sich aus dem Grundgesetz keine weiteren zeitlichen Vorgaben für Gesetzgebungsverfahren. Auch die Geschäftsordnung des Bundestages trifft nur wenige Aussagen zu Fristen beim Gesetzgebungsverfahren. Sie schreibt lediglich vor, dass die zweite Beratung erst am zweiten Tag nach Verteilung der Ausschussempfehlungen und des Ausschussberichts erfolgen darf (Ausnahmen können mit einer Zweidrittelmehrheit oder bei als dringlich erklärten Gesetzen mit der Kanzlermehrheit beschlossen werden, \81 GOBT).

15 Vgl. für das Folgende: Brun-Otto Bryde, \$30. Stationen, Entscheidungen und Beteiligte im Gesetzgebungsverfahren, in: Hans-Peter Schneider / Wolfgang Zeh (Hrsg.), Parlamentsrecht und Parlamentspraxis in der Bundesrepublik Deutschland, Berlin / New York 1989, S. 858 - 881; Wolfgang Ismayr, Der Deutsche Bundestag, Opladen 2000, S. 239 - 298; Hans Schneider, Gesetzgebung. Ein Lehr- und Handbuch, 3. Auflage, Heidelberg 2002, S. 56 - 117.

16 Im Jahre 1968 wurde die Frist, in der der Bundesrat seine Stellungnahme abgeben muss, von drei auf sechs Wochen erhöht sowie die Möglichkeit geschaffen, als besonders eilbedürftig bezeichnete Vorlagen der Bundesregierung ohne Stellungnahme des Bundesrates in den Bundestag einzubringen, Peter Schindler, a.a.O., S. 2969. 
Die Zweitagesfrist gilt auch für die dritte Beratung, wenn Änderungen am Gesetzentwurf beschlossen wurden ( $\$ 84$ GOBT).

(3) Verfahren im Bundesrat, Vermittlungsausschuss, „4. und 5. Lesung“: Stärker reglementiert und mit Maximalfristen versehen ist der dritte Teil des Gesetzgebungsverfahrens, der folgende Schritte umfassen kann: (a) die Behandlung des Entwurfs im Bundesrat (Art. 77 Abs. 2 und 78 GG), (b) das Vermittlungsverfahren (Art. 77 Abs. 2 GG), (c) die Abstimmung über einen möglichen Vorschlag des Vermittlungsausschusses im Bundestag („4. Lesung“, Art. 77 Abs. 2 GG), (d) die Zustimmung (respektive die Verweigerung der Zustimmung) des Bundesrats zum Gesetz, (e) die Einlegung eines Einspruchs durch den Bundesrat und gegebenenfalls (f) die Zurückweisung des Einspruchs durch den Bundestag in einer so genannten 5. Lesung. Der Bundesrat kann bei Einspruchsgesetzen innerhalb von drei Wochen den Vermittlungsausschuss anrufen (bei Zustimmungsgesetzen können dies bekanntlich auch die Bundesregierung und der Bundestag), und er hat bei Zustimmungsgesetzen in „angemessener Frist“ Beschluss zu fassen (Art. 77 Abs. 2a GG). Nach Ende des Vermittlungsverfahrens kann der Bundesrat innerhalb von zwei Wochen Einspruch gegen ein Gesetz einlegen.

(4) Gegenzeichnung, Ausfertigung und Verkündung: Ein Gesetz kommt zustande, wenn der Bundesrat ihm zustimmt, der Bundestag den Vermittlungsvorschlag annimmt oder einen Einspruch des Bundesrates zurückweist (Art. 78 GG). Um in Kraft treten zu können, bedarf das Gesetz noch der Gegenzeichnung durch die Bundesregierung, der Ausfertigung durch den Bundespräsidenten und der Verkündung im Gesetzblatt (Art. 82 GG). Die Einhaltung von Fristen schreibt das GG hier nicht vor, doch wird man unterstellen dürfen, dass alle diese Schritte „unverzüglich“ zu erfolgen haben. ${ }^{17}$

In der weiteren Analyse werden lediglich drei Verfahrensschritte unterschieden, neben dem Vorverfahren und dem Verfahren im Bundestag werden die beiden letzten Stadien, die sich an den Gesetzesbeschluss im Bundestag anschließen, der Einfachheit halber unter der Überschrift „post-parlamentarisches Verfahren “ 18 zusammengefasst. Wenn nicht anders angegeben, bezieht sich „Dauer von Gesetzgebungsverfahren“ auf die Tage zwischen Einbringung der Gesetzesinitiative in den Bundestag bis zur Verkündung, schließt also Vorverfahren aus.

Insgesamt sind die rechtlichen Vorgaben, die ein Verfahren zeitlich begrenzen und strukturieren könnten, rudimentär. Anders als etwa im britischen Parlament, dessen Legislaturperiode in "Sessions“ mit eigenständigem Diskontinuitätsprinzip unterteilt ist, kann sich ein Gesetzgebungsverfahren in Deutschland sowohl über wenige Wochen als auch über die volle Legislaturperiode erstrecken. Die zeitliche Strukturlosigkeit der parlamentarischen Prozedur manifestiert sich prägnant in ihrer minimalen und maximalen Dauer (vgl. Tabelle 3).

17 Wolfgang Ismayr, a.a.O., S. 290.

18 Mit dem Begriff werden lediglich die beiden letzten Stadien im Gesetzgebungsverfahren beschreibend zusammengefasst. Er hat keinen erklärenden Gehalt und bezieht sich nicht auf Theorien über „postparlamentarische Demokratien“, vgl. für viele: Arthur Benz, Postparlamentarische Demokratie? Demokratische Legitimation im kooperativen Staat, in: Michael Greven (Hrsg.), Demokratie - eine Kultur des Westens?, 20. Wissenschaftlicher Kongreß der Deutschen Vereinigung für Politische Wissenschaft, Opladen 1998, S. 201 - 222. 


\begin{tabular}{|c|c|c|c|}
\hline \multicolumn{4}{|c|}{$\begin{array}{l}\text { Tabelle 3: Durchschnittliche, minimale und maximale Dauer von Gesetzgebungsverfahren } \\
(9 ., 10 ., 11 ., 12 ., \text { und 15. Wahlperiode des Bundestages) }\end{array}$} \\
\hline & \multicolumn{3}{|c|}{$\begin{array}{l}\text { Dauer (in Tagen) zwischen Einbringung in Bundestag } \\
\text { und Verkündung des Gesetzes }\end{array}$} \\
\hline & $\begin{array}{l}\text { durchschnittliche } \\
\text { Dauer }\end{array}$ & $\begin{array}{l}\text { minimale } \\
\text { Dauer }\end{array}$ & $\begin{array}{c}\text { maximale } \\
\text { Dauer }\end{array}$ \\
\hline $1980-1983$ & 187 & 23 & 665 \\
\hline 1983-1987 & 259 & 38 & 1261 \\
\hline $1987-1990$ & 212 & 8 & 1292 \\
\hline 1990-1994 & 211 & 21 & 1236 \\
\hline $2002-2005$ & 163 & 33 & 691 \\
\hline
\end{tabular}

Gleichwohl: Die durchschnittliche Dauer von Gesetzgebung weist ein recht stabiles Muster auf (vgl. Tabelle 4). In der Regel brauchte ein Gesetz - ohne Vorverfahren - in den bisher beendeten Wahlperioden durchschnittlich zwischen sieben und acht Monate (220 Tage), um in den Bundestag eingebracht und verkündet zu werden. Davon entfielen circa 65 Tage auf die beiden letzten Stadien nach der dritten Lesung im Bundestag, also auf das post-parlamentarische Verfahren. Deutlich schneller mit 187 beziehungsweise 163 Tagen ging es jedoch in den beiden verkürzten Wahlperioden 1980 bis 1983 und 2002 bis 2005 (jedoch nicht in der ebenfalls verkürzten sechsten WP); mit durchschnittlich 266 Tagen am längsten brauchte ein Gesetz in der siebten Wahlperiode (1972 bis 1976).

\section{Dauer von Gesetzgebungsverfahren in der 15. Wahlperiode (2002 bis 2005)}

Wodurch wird die Dauer von Gesetzgebungsverfahren insgesamt bestimmt? Die Zusammenhänge sind keineswegs so eindeutig, wie dies in der politischen, aber auch teilweise in der fachwissenschaftlichen Debatte bisweilen unterstellt wird. Schon eine oberflächliche Betrachtung der Entwicklung seit 1949 relativiert häufig vorgebrachte Annahmen (vgl. Tabelle 4):

(1) Zuerst und wider Erwarten ist festzuhalten, dass die Anzahl von Vermittlungsverfahren nur schwach korreliert mit der Dauer von Gesetzgebungsverfahren. Besonders prägnant zeigt sich diese Entkopplung in der 4. und 10. Wahlperiode, in denen der Vermittlungsausschuss lediglich 39- beziehungsweise 4-mal angerufen wurde, ein Gesetz aber nach seiner Einbringung in den Bundestag mit 254 beziehungsweise 259 Tagen überdurchschnittlich lange auf seine Verkündung warten musste. Genau der konträre Zusammenhang - eine relativ hohe Anzahl von Vermittlungsverfahren und eine unterdurchschnittliche Dauer von Gesetzgebungsverfahren - zeigte sich in der 1. und 15. Wahlperiode.

(2) Der Anteil der Zustimmungsgesetze einerseits und die Dauer von Gesetzgebungsverfahren andererseits korrelierten mit einem Wert von 0,51 stark positiv. Allerdings lagen die Anteile zustimmungsplichtiger Gesetze in der 11. und 12. Wahlperiode mit 55,2 beziehungsweise 56,6 Prozent relativ hoch, in diesen Perioden dauerten Gesetzgebungsverfahren 


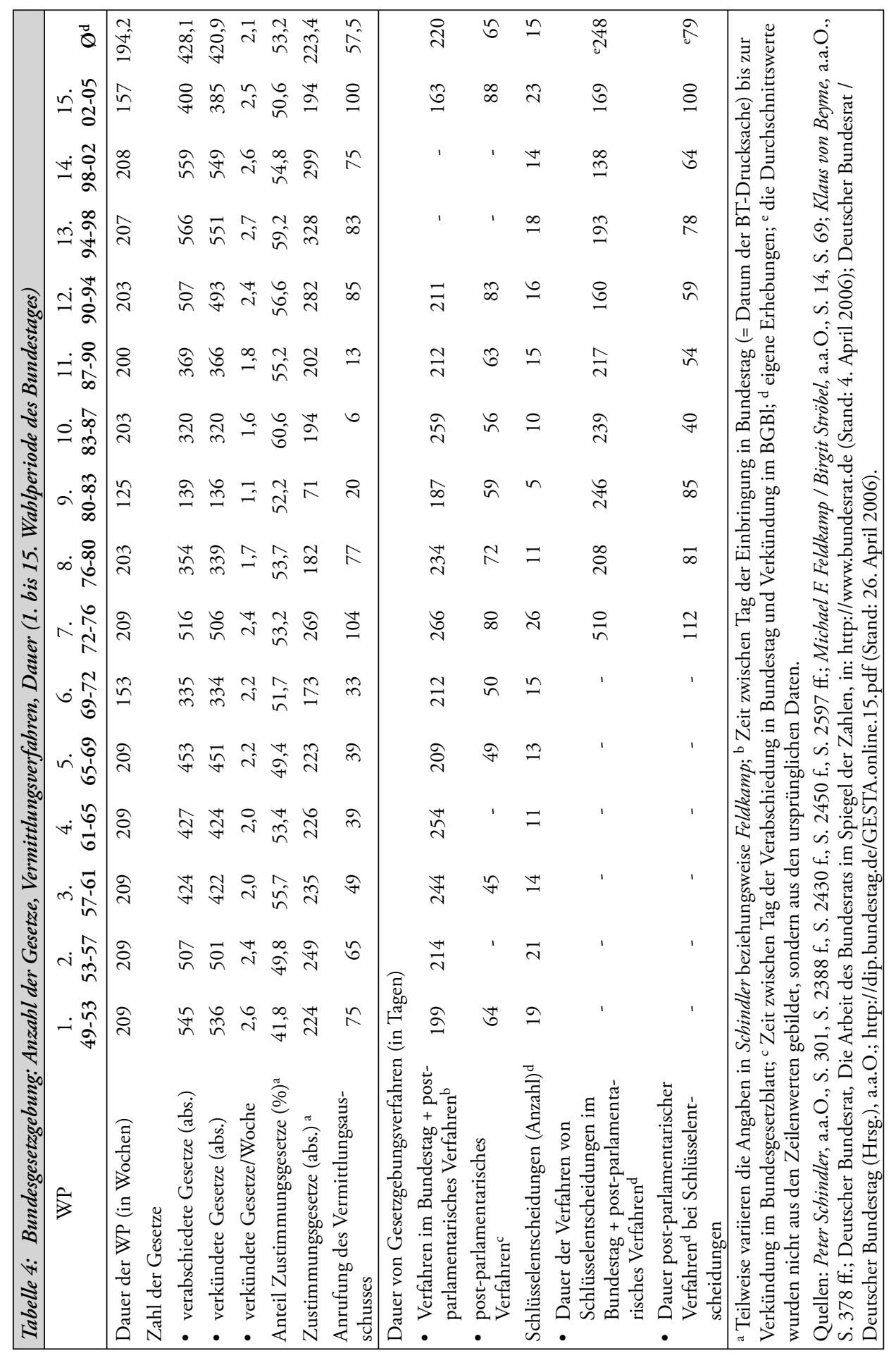


aber mit 212 beziehungsweise 211 Tagen jedoch unterdurchschnittlich lange. Doch ändern diese statistischen Ausreißer wenig an dem grundsätzlichen Zusammenhang, obschon sie anzeigen, dass es hier noch weitere intervenierende Faktoren geben kann, die die Verfahrensdauer beeinflussen.

(3) Bestätigt wird schließlich die verbreitete Vermutung, dass sich eine hohe Anzahl von Vermittlungsverfahren auf die Dauer der post-parlamentarischen Verfahren verlängernd auswirkt. Anders formuliert: Je mehr Vermittlungsverfahren stattfanden, desto mehr Zeit verstrich zwischen der 3. Lesung im Bundestag und der Verkündung eines Gesetzes im Bundesgesetzblatt. Das kann nicht überraschen.

Es versteht sich, dass diese Befunde vorsichtig zu interpretieren sind. Doch zumindest werfen sie die Frage auf, ob und inwieweit der Beteiligungsföderalismus die Ursache ist für die beklagte Langwierigkeit von Gesetzgebungsverfahren in Deutschland.

Teilweise finden sich die genannten Zusammenhänge in der 15. Wahlperiode bestätigt: Der mit 50,6 Prozent vergleichsweise geringe Anteil zustimmungspflichtiger Gesetze ging zwischen 2002 und 2005 einher mit relativ kurzen Verfahren. Gleichzeitig hat die hohe Anzahl von Vermittlungsverfahren das post-parlamentarische Verfahren auf durchschnittlich 88 Tage verlängert (vgl. Tabelle 4).

Das Profil der Gesetzgebung der 15. Wahlperiode weicht allerdings in wichtigen Aspekten von denjenigen früherer Wahlperioden ab. So fiel die Anzahl der verkündeten Gesetze - trotz der verkürzten Wahlperiode - mit 385 höher aus als in vielen „normalen“ Wahlperioden (zum Beispiel 6., 8., 9., 10. oder 11.). Der Bundestag war in der 15. Wahlperiode mit 2,5 verkündeten Gesetzen pro Woche folglich überdurchschnittlich aktiv, auch wenn dies leicht unter dem Niveau der 13. und 14. Wahlperiode lag. Dieser legislative Aktivismus in der 15. Wahlperiode war zudem von einer hohen Konfliktintensität begleitet, zumindest gemessen an der Anzahl der Vermittlungsverfahren: Mit 100 liegt die 15. Wahlperiode vor der 12., 13. und 14. und nur knapp hinter der 7., wobei zu beachten ist, dass zwischen 2002 und 2005121 Gesetze weniger verkündet wurden als zwischen 1972 und 1976. Doch trotz der hohen Anzahl von Vermittlungsverfahren sank in der 15. Wahlperiode die Gesamtdauer bei der Gesetzgebung auf 163 Tage, den niedrigsten Wert seit 1949. Lediglich in der ebenfalls verkürzten 9. Wahlperiode brauchte ein Gesetz mit 187 Tagen ähnlich lange. Es ist daher zu vermuten, dass bei einem vorzeitigen Ende der Legislatur-periode gerade jene Gesetze dem Diskontinuitätsprinzip zum Opfer fallen, die besonders langwierig sind. (Jedoch gilt dies allenfalls bedingt für die ebenfalls verkürzte 6. Wahlperiode.)

Unterteilt man die Verfahren in die oben genannten Stadien, ergibt sich für die 15. Wahlperiode die in Tabelle 5 aufgewiesene Struktur:

(1) Alle Gesetze: Abgesehen von der mit 163 Tagen vergleichsweise kurzen Gesamtdauer weist diese Wahlperiode noch eine weitere Besonderheit auf: Die Beratungen im Bundestag nahmen mit 75 Tagen weniger Zeit in Anspruch als die post-parlamentarischen Verfahren (88 Tage).

(2) Zustimmungs- und Einspruchsgesetze: Auffällig ist zudem, dass sich in der 15. Wahlperiode die Verfahrensdauer zwischen Zustimmungs- und Einspruchsgesetzen allenfalls marginal unterschied. Zustimmungsgesetze benötigten im Durchschnitt zehn Tage mehr (168 statt 158) als Einspruchsgesetze - bei Gesetzen mit Vorverfahren, waren es 15 Tage (234 statt 219). Das dürfte als eine zu vernachlässigende Verzögerung gelten. 


\begin{tabular}{|c|c|c|c|c|c|}
\hline \multicolumn{6}{|l|}{ Tabelle 5: } \\
\hline & \multirow[t]{2}{*}{$\begin{array}{c}\text { alle } \\
\text { Gesetze }\end{array}$} & \multicolumn{2}{|c|}{$\begin{array}{l}\text { Zustimmung } \\
\text { erforderlich }\end{array}$} & \multicolumn{2}{|c|}{$\begin{array}{l}\text { Vermittlungs- } \\
\text { verfahren }\end{array}$} \\
\hline & & $\mathrm{Ja}$ & Nein & $\mathrm{Ja}$ & Nein \\
\hline Anzahl der verkündeten Gesetze & 385 & 195 & 190 & 87 & 298 \\
\hline \multicolumn{6}{|c|}{ Dauer von Gesetzgebungsverfahren (in Tagen) } \\
\hline (1) Vorverfahren & 55 & 58 & 51 & 53 & 55 \\
\hline (2) Beratungen Bundestag & 75 & 76 & 74 & 71 & 76 \\
\hline (3) post-parlamentarisches Verfahren & 88 & 92 & 84 & 119 & 79 \\
\hline$(2)+(3)$ & 163 & 168 & 158 & 190 & 155 \\
\hline$(1)+(2)+(3)$ & a227 & ${ }^{\mathrm{a}} 234$ & a219 & a263 & ${ }^{\mathrm{a}} 218$ \\
\hline \multicolumn{6}{|c|}{$\begin{array}{l}\text { a Bezieht nur Gesetze ein, die das Vorverfahren durchlaufen haben, andere Gesetze sind nicht berück- } \\
\text { sichtigt. Der Wert stellt also nicht die Spaltensumme dar, sondern wurde auf Grundlage der Ursprungs- } \\
\text { daten eigenständig berechnet. } \\
\text { Quelle: http://dip.bundestag.de/GESTA.online.15.pdf (Stand: 26. April 2006), eigene Auszählung. }\end{array}$} \\
\hline
\end{tabular}

(3) Vermittlungsverfahren (vgl. Tabelle 6): Wie erwähnt, wurde in der 15. Wahlperiode bei 100 Gesetzen der Vermittlungsausschuss angerufen, 90-mal durch den Bundesrat, elfmal durch die Bundesregierung und eine Anrufung ging auf den Bundestag zurück. ${ }^{19}$ Erstaunlich ist dabei die hohe Anzahl von Vermittlungsverfahren bei Einspruchsgesetzen (45 von 190), unbeschadet des fehlenden absoluten Vetorechts des Bundesrates in diesen Fällen (Tabelle 6). Dabei verlängerte die Vermittlungsprozedur das Gesamtverfahren im

\begin{tabular}{|c|c|c|c|}
\hline \multicolumn{4}{|c|}{$\begin{array}{l}\text { Vermittlungsverfahren und Dauer von Gesetzgebungsverfahren bei Einspruchs- } \\
\text { und Zustimmungsgesetzen (15. Wahlperiode des Bundestages) })^{a}\end{array}$} \\
\hline & $\begin{array}{l}\text { mit Vermittlungs- } \\
\text { verfahren }\end{array}$ & $\begin{array}{c}\text { ohne Vermittlungs- } \\
\text { verfahren }\end{array}$ & gesamt \\
\hline \multicolumn{4}{|l|}{ Anzahl (abs.) } \\
\hline - bei Einspruchsgesetzen & 45 & 145 & 190 \\
\hline - bei Zustimmungsgesetzen & 42 & 153 & 195 \\
\hline - gesamt (abs.) & 87 & 298 & 385 \\
\hline \multicolumn{4}{|c|}{ Dauer der Gesetzgebungsverfahren (in Tagen) } \\
\hline - bei Einspruchsgesetzen & 186 & 149 & 158 \\
\hline - bei Zustimmungsgesetzen & 195 & 160 & 168 \\
\hline - gesamt & 190 & 155 & 163 \\
\hline
\end{tabular}

19 Die Differenz zu den in Tabelle 6 aufgeführten 87 Anrufungen des Vermittlungsausschusses ist auf den Umstand zurückzuführen, dass hier nur die verkündeten Gesetze gezählt wurden, während in die Statistik der Bundestagsverwaltung auch Vermittlungsverfahren bei nicht verkündeten Gesetzen eingingen (wegen Verweigerung der Zustimmung, wegen Ende der Legislaturperiode etc.). 
Durchschnitt um circa einen vollen Monat (190 statt 155 Tage). Das ist - bezogen auf den Gesamtprozess - eine beachtliche Verzögerung, dennoch dürfte auch dieser Wert kaum ein ausreichender Grund dafür sein, parlamentarische Entscheidungsprozesse insgesamt als langwierig und schleppend zu disqualifizieren. Zudem scheint vor diesem Hintergrund die Hoffnung, mit der Reduzierung des Anteils zustimmungspflichtiger Gesetze könnte die parlamentarische Entscheidung beschleunigt werden, doch recht trügerisch.

Schließlich ist noch auf die Rolle des Vorverfahrens einzugehen, also die Behandlung von Gesetzentwürfen im Bundesrat, bevor der Gesetzgebungsprozess im Bundestag überhaupt begonnen hat. Bemerkenswert ist, dass Gesetze, die ein Vorverfahren durchlaufen, auch in späteren Entscheidungsstadien häufig mehr Zeit benötigen. So dauerte es bei Vorlagen der Bundesregierung (oder aus der Mitte des Bundesrates) in der 15. Wahlperiode im Durchschnitt 227 Tage bis zur Verkündung, während Gesetze ohne Vorverfahren lediglich 129 Tage benötigten. Verursacht wurde diese Differenz keineswegs nur durch die Vorverfahren selbst, die durchschnittlich 55 Tage in Anspruch nahmen, sondern auch durch den Umstand, dass die Beratungen im Bundestag und die post-parlamentarischen Verfahren länger dauerten als bei Gesetzen ohne Vorverfahren. ${ }^{20}$ Die Ursachen für diese Differenz sind auf Grundlage der Daten nicht zu ermitteln. Es kann also nur spekuliert werden. Dabei spricht einiges für die Vermutung, dass durch die Stellungnahmen, die im Vorverfahren abgegeben werden, Entscheidungsprozesse inhaltlich aufgeladen werden. Die in den Stellungnahmen des Bundesrates (beziehungsweise der Bundesregierung) aufgeführten Punkte müssen mithin im nachfolgenden Gesetzgebungsprozess zeitintensiv abgearbeitet werden.

\section{Dauer von Gesetzgebungsverfahren bei Schlüsselentscheidungen}

Die Merkmale, die für die 15. Wahlperiode gelten, finden sich im Wesentlichen durch die Analyse der 138 Schlüsselentscheidungen bestätigt - allerdings mit bemerkenswerten Niveauunterschieden. So erforderten fast zwei Drittel (62,3 Prozent) aller Schlüsselentscheidungen die Zustimmung des Bundesrates, und knapp die Hälfte (44,2 Prozent) durchlief ein Vermittlungsverfahren, wovon wiederum allein 35 (von 61) auf die beiden verkürzten Wahlperioden entfielen. Durchschnittlich dauerte ein Gesetzgebungsverfahren bei Schlüsselentscheidungen 248 Tage, also circa einen Monat länger als ein „normales“ Gesetz. Doch schließt dies die siebte Wahlperiode ein, in der eine Schlüsselentscheidung rund 510 Tage brauchte, um als Gesetz verkündet zu werden. Wird diese außergewöhnliche Wahlperiode ausgeschlossen, dauerte ein Verfahren für solch politisch zumeist umstrittene Neuregelungen mit 187 Tagen sogar weniger lang als ein normales Gesetz mit 220 Tagen. Es kann also nicht davon gesprochen werden, dass die parlamentarische Behandlung von Schlüsselentscheidungen besonders langwierig wäre (vgl. Tabelle 7).

20 Bei Gesetzen mit Vorverfahren dauerte das Verfahren im Bundestag durchschnittlich 79 Tage und das post-parlamentarische Verfahren 92 Tage; bei Gesetzen ohne Vorverfahren waren es 58 beziehungsweise 71 Tage. 


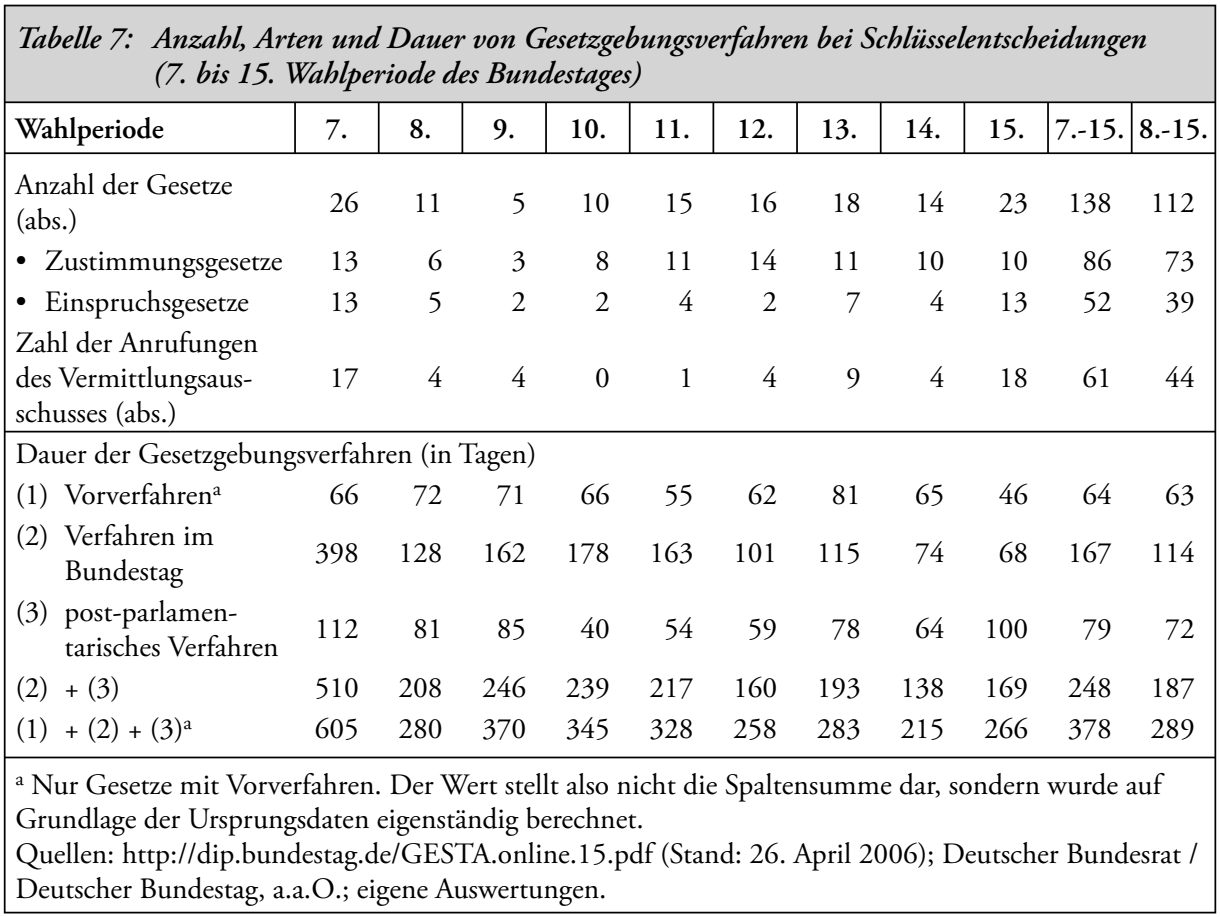

Wie in der 15. WP war auch bei Schlüsselentscheidungen für die Dauer von Gesetzgebungsverfahren weniger der Umstand von Bedeutung, ob es sich um Zustimmungsgesetze handelte, die durchschnittlich fünf Tage mehr benötigten als Einspruchsgesetze (250 statt 245 Tage) (vgl. Tabelle 8). Vielmehr waren es vor allem Vermittlungsverfahren, die die Gesetzgebung bei Schlüsselentscheidungen um recht beachtliche drei Monate (305 statt 203 Tage) verzögerten (vgl. Tabelle 8). Besonders dramatisch wirkte sich dieser Zusammenhang

\begin{tabular}{|c|c|c|c|}
\hline \multicolumn{4}{|c|}{$\begin{array}{l}\text { Schlïsselentscheidungen: Anzahl und Dauer, unterteilt nach Einspruchs- und } \\
\text { Zustimmungsgesetzen sowie nach Vermittlungsverfahren (7. bis 15. WP des } \\
\text { Bundestages) }\end{array}$} \\
\hline & $\begin{array}{l}\text { ohne Vermittlungs- } \\
\text { verfahren }\end{array}$ & $\begin{array}{l}\text { mit Vermittlungs- } \\
\text { verfahren }\end{array}$ & gesamt \\
\hline \multicolumn{4}{|l|}{ Anzahl der Gesetze } \\
\hline - Einspruchsgesetze (abs.) & 25 & 27 & 52 \\
\hline - Zustimmungsgesetze (abs.) & 52 & 34 & 86 \\
\hline - gesamt (abs.) & 77 & 61 & 138 \\
\hline \multicolumn{4}{|l|}{ Dauer der Verfahren (in Tagen) } \\
\hline - bei Einspruchsgesetzen & 227 & 262 & 245 \\
\hline - bei Zustimmungsgesetzen & 192 & 339 & 250 \\
\hline - bei allen & 203 & 305 & 248 \\
\hline
\end{tabular}


bei Zustimmungsgesetzen aus; bei diesen verzögerte ein Vermittlungsverfahren die Verkündung um 147 Tage (339 statt 192 Tage).

Ebenso prägnant fallen die Unterschiede aus, wenn die Schlüsselentscheidungen nach Vorverfahren differenziert werden. Bei solchen Gesetzen benötigten Vorlagen der Regierung beziehungsweise des Bundesrates über ein Jahr (378 Tage), während Gesetzesinitiativen aus der Mitte des Bundestages schon nach 152 Tagen verkündet wurden. Überraschend ist dabei allerdings, dass die Verfahren im Bundestag mit 223 Tagen (statt 86 Tagen) außerordentlich viel Zeit in Anspruch nahmen. Diese Differenz verkürzt sich zwar beträchtlich, wenn die 7. WP unberücksichtigt bleibt, doch wurde auch in der 8. bis 15 . Wahlperiode eine Schlüsselentscheidung, die ein Vorverfahren durchlief, im Bundestag circa zwei Monate länger beraten als Gesetzentwürfe aus der Mitte des Parlaments (144 statt 80 Tage). Auch hier lassen die Daten allenfalls die bereits genannten Vermutungen zu. Durch die Stellungnahmen, die im Rahmen von Vorverfahren dem Gesetzentwurf angefügt werden, dürfte in Bundestag und Bundesrat zusätzlicher Beratungs- und Verhandlungsbedarf entstehen, was die Verfahren insgesamt verlängert.

\section{Schneller nicht gleich besser! Oder: Zum Unterschied zwischen politischer Entscheidungs- findung und parlamentarischer Gesetzgebung}

Die empirische Bestandsaufnahme lässt vier Schlussfolgerungen zu:

(1) Die Analyse der zeitlichen Struktur des Gesetzgebungsverfahrens im Bund zeigt, dass die Annahme, ein hoher Anteil zustimmungspflichtiger Gesetze führe zu Verzögerungen im Gesetzgebungsprozess, zu kurz greift, sogar unzutreffend scheint. Verfahrensverlängernd wirkt nicht die Zustimmungspflichtigkeit an sich; vielmehr sind es die Anrufung des Vermittlungsausschusses sowie Vorverfahren, die Gesetzgebungsprozesse zeitlich strecken. Keiner dieser Aspekte ist durch die Reformen 2006 geändert worden.

(2) Schlüsselentscheidungen dauerten keineswegs wesentlich länger als „normale“ Gesetze, auch wenn sie überdurchschnittlich häufig zustimmungspflichtig waren. Die Konfliktintensität bei politisch umstrittenen Entscheidungen hat sich also nicht in Entscheidungsverzögerungen niedergeschlagen, sondern in einer vergleichsweise hohen Anzahl von Anrufungen des Vermittlungsausschusses: So wurden von 138 Schlüsselentscheidungen 61 im Vermittlungsausschuss behandelt, also 44,2 Prozent, während dies bei allen Gesetzen zwischen der 7. bis 15. Wahlperiode „lediglich“ 15,4 Prozent waren (563 von 3.645).

(3) Die Klage über langwierige Entscheidungsprozesse kann im parlamentarischen Verfahren keinen überzeugenden Grund finden. Vielmehr nährt sie sich aus den vor- und außerparlamentarischen Debatten und Diskussionen, die politische Entscheidungsfindungen begleiten - und wohl auch begleiten sollten. Die Klagen richten sich also gegen den „deliberativen“ Teil von Rechtssetzung und mobilisieren einen „latenten Dezisionismus“. „Es gibt in Deutschland“, so Angela Merkel, „eine große Sehnsucht, die großen Probleme mit einem einzigen großen Wurf zu beheben. ${ }^{21}$

21 „Wir muten dem Bürger eine ganze Menge zu“. Angela Merkel verteidigt die finanziellen Belastungen und kündigt weitere Reformen an, in: Süddeutsche Zeitung vom 20./21. Mai 2006, S. 6 . 
(4) Ein ideales Maß von Gesetzgebungsdauer existiert nicht und lässt sich abstrakt auch nicht festlegen. Es trifft zu, dass Gesetzgebungsverfahren in Deutschland vergleichsweise lange dauern. In Großbritannien benötigten die in der Session 2004/05 verabschiedeten Gesetze rund 113 Tage; in Bayern brauchte ein Gesetz von der Einbringung bis zur Verkündung in der 14. Wahlperiode (1998 bis 2003) circa 137 Tage. ${ }^{22}$ Doch taugen solche Vergleiche keineswegs dazu, normative Standards zu bilden. Kurze oder kürzere Gesetzgebungsverfahren als in Deutschland sind nicht per se die besseren.

22 Eigene Auszählungen auf Grundlage von: Bayerischer Landtag, Tätigkeitsbericht über die 14. Wahlperiode 1998/2003, München, http://www.landtag-bayern.de/pdf_internet/Taetigkeitsbericht_14WP.pdf (Stand: 13. Mai 2006) sowie dem Sessional Information Digest des House of Commons, http://www.publications.parliament.uk/pa/cm/cmsid.htm (Stand 10. April 2006). Das Gesetzgebungsverfahren in Großbritannien beginnt mit der ersten Lesung und endet mit dem Royal Assent, in Bayern wird das Verfahren begrenzt durch den Tag der Einbringung eines Gesetzentwurfs in den Landtag (Drucksachendatum) und dem Tag der Verkündung des Gesetzes.

\section{Private Bills in angelsächsischen Regierungssystemen: Legitimitätsressource oder Unterlaufen der Gewaltenteilung?}

\section{Thomas Krumm}

Anders als in der Rechtswissenschaft ${ }^{1}$ ist das in den angelsächsisch geprägten Regierungssystemen verbreitete Gesetzgebungsverfahren der Private Bills von der deutschen Politikwissenschaft bislang fast überhaupt nicht wahrgenommen worden. Private Bills eröffnen einzelnen privaten Akteuren und Interessen die Möglichkeit, Gesetzgebung durch einzelne Abgeordnete, die als parlamentarische „Paten“ fungieren, zu initiieren, die sich explizit zugunsten dieser privaten Akteure auswirkt. Solche Private Bills sind nicht zu verwechseln mit Private Members' Bills, dem Recht einzelner Abgeordneter, im Bereich der allgemeinen Public Bills initiativ zu werden. Private Bills können, im Unterschied zu den Public Bills, einzelnen Individuen bestimmte Vorteile gewähren wie etwa Ausnahmen von den allgemeinen Gesetzen. In der Bundesrepublik Deutschland würde man diesen Gesetzestyp als Einzelfall- und Maßnahmengesetz bezeichnen. Absicht der folgenden Untersuchung ist eine vergleichende Einführung in die „private“ Gesetzgebung in Großbritannien, Schottland, den USA und Kanada sowie eine Gegenüberstellung mit den in der Bundesrepublik Deutschland stark eingeschränkten, aber nicht grundsätzlich verbotenen Einzelfall- beziehungsweise Maßnahmengesetzen.

1 Grundlegend nach wie vor Julius Hatschek, Englisches Staatsrecht, Tübingen 1905, Band 1, S. $120 \mathrm{ff}$. und S. 503 ff., Band 2, S. 376 f. und passim. 